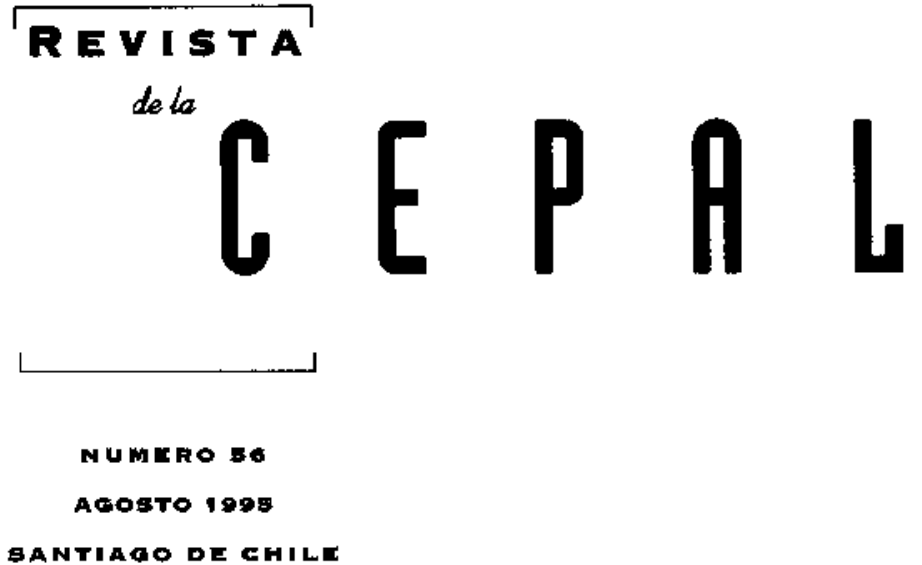

ANIBAL PINTO

Director

EUGENIO LAHERA

Secretario Técnico

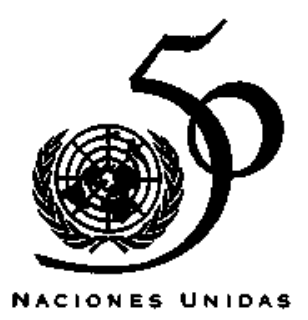


Democracla y desarrollo

Fernando H. Cardoso

¿Es posible crecer con equidad?

Joseph Ramos

Estabilidad y estructura: Interacclones en el crecimiento económlco

Jose Maria Fanelli y Roberto Frenkel

Reforma a los sistemas de pensiones en América Latina

Andras Uthoff

Tendenclas económicas en China: significado para el comerclo con América Latina y el Caribe

Mikio Kuwayama

El Intercamblo económico entre América Latina y las

economias dinámicas de Asla

Ronald Sprout

La relación económica entre la Amórica Latina y la Unlón Europea

Roberto Smith Perera

Nuevas implicaclones de las reglas do orlgen

Eduardo Gitli

Globalización y reestructuración energética en Amórlca Latina

Fernando Sánchez Albavera

El caloidoscoplo de la competitlvidad

Geraldo Muller

La privatización de los servicios públicos del agua

Miguel Solanes

¿Cuénto se puede gastar en educaclón?

Guillermo Labarca

Mujeres y migranteo: deslgualdades en el

mercado laboral de Santlago de Chile

Ivonne Szasz

Orlentaciones para los colaboradores de la Rovista de la CEPAL 


\section{El caleidoscopio de la competitividad}

\section{Ceraldo Müller}

Investigador,

Universidad Estadual Paulista (UNESP). Miembro del Grupo Ejecutivo de la UNESP para el Mercosur (PROMERCO)
La competitividad se ha convertido en una de las principales "normas" del inestable juego internacional. La apertura comercial, los ajustes estructurales, la reconversión productiva, la coexistencia inteligente con los recursos naturales, la lucha contra la pobreza, se enfocan de una manera u otra a través del prisma de la competitividad. Lo que la ha transformado en una especie de principio obligado de evaluación internacional, que influye en la formulación y aplicación de estrategias empresariales y políticas nacionales. La literatura sobre competitividad entrega un abanico de definiciones, que van desde las centradas en los aspectos económicos hasta las que intentan articular los aspectos tecnoeconómicos, sociopolíticos y culturales del proceso competitivo. Las diferencias surgen de la manera de examinar las relaciones entre desarrollo y competitividad. El autor postula que se puede contar con un mapa de la competitividad, una red de conceptos claves articulados cuyo objetivo es el mismo cualquiera sea la definición de la competitividad: conquistar, mantener y ampliar la participación en los mercados. Ese mapa muestra un conjunto ordenado y flexible de conceptos - un territorio y sus caminos- que se puede adaptar a los intereses y objetivos de quien lo utilice, y que, igual que un caleidoscopio, sirve para innumerables propósitos y frente a problemas particulares permite elaborar el concepto adecuado. 


\section{Concepto y mapa}

Hay palabras que adquieren el don de ser extremadamente precisas, específicas, y a la vez extremadamente genéricas, sin límites; altamente operativas y mensurables, y a la vez sumamente abstractas y extensas. Sin embargo, en cualquiera de los casos, tienen el privilegio de forjar conductas y perspectivas, y como herramientas de evaluación, de influir en la vida práctica. Una de estas palabras mágicas es "competitividad". Quizás una manera de comprender la magia de esta palabra es distinguirla de otra: "competencia".

Se puede entender la competencia como parte de la lucha económica, y la capacidad para la competencia como el proceso que desemboca en la rivalidad entre los gnupos de vendedores. Se podría, por lo tanto, distinguir la competencia de la competitividad si consideramos esta última como el conjunto de habilidades y condiciones requeridas para el ejercicio de la competencia. La competencia sería el resultado de la competitividad y estaría incluida en ella.

Esto podría ser una explicación válida si no existiera una perspectiva globalizadora del resultado y del proceso. Pues se puede entender la competencia como un proceso de enfrentamiento de varios capitales, o sea, de las unidades de poder de valorización y de expansión economica que la propiedad del capital confiere. La competencia debe ser considerada "como parte integral, inseparable del movimiento global de acumulación de capital"; es, por lo tanto, "el motor básico de la dinámica capitalista" (Possas, 1985). Además, el enfrentamiento de capitales ocurre en el mercado, definido como el lugar de la competencia capitalista.

Competencia y competitividad serían, desde esta perspectiva, intercambiables: una cuestión de preferencia. Y la palabra competitividad no sería más que un término en boga. $O$ como la define un diccionario español de economía: "competitividad - cacofonico término introducido en los años 80, por capacidad para competir, especialmente en los mercados exte- riores. En inglés: competitiveness"; lo cual sugiere que se trata de un término exclusivamente económico, equivalente a competencia (Tamanes, 1988).

Aunque se puede entender que el movimiento global de acumulación de capital incluye todas las esferas de la sociedad capitalista, y de esta manera estaría resuelta la cuestión que queremos tratar, faltaría tener una visión integrada de la competencia para entender las nuevas interrogantes abordadas por varios autores contemporáneos, que consideran la competitividad desde una perspectiva singular.

Se puede separar con precisión competencia y competitividad, o juntarlas por medio de la acumulación global de capital, lo que podría ayudar bastante para entender la polisemia que actualmente caracteriza la palabra competitividad, si hubiera este cuadro de referencia. En todo caso, después de examinar parte de la voluminosa bibliografía disponible sobre el tema, se concluye que vale la pena presentar algunas de las distintas conceptualizaciones existentes, señalar sus connotaciones y contrastarlas con los hechos. Creemos que con lo que en realidad se puede contar es con un mapa de la competitividad, y no con un determinado concepto de ella con validez general.

La competitividad puede entenderse mejor mediante un mapa, o una red de conceptos articulados, cuyo objetivo es el mismo con cualquier definición del término: conquistar, mantener y ampliar la participación en los mercados. Este mapa se asemeja a un caleidoscopio; a un conjunto ordenado y flexible de conceptos que puede adaptarse a los intereses y objetivos de las personas que desean utilizarlo. Y, como un caleidoscopio, el mapa sirve para innumerables fines: presenta un territorio y sus caminos, y las incógnitas que acompañan los caminos parcialmente conocidos, pero sin proveer los elementos específicos para el examen de problemas particulares. Para ellos hay que elaborar el concepto adecuado. $\square$ El autor agradece al Instituto Interamericano de Cooperación para la Agricultura (IICA) (San José de Costa Rica) la oportunidad que le brindó de estudiar el tema de la competitividad en el periodo 1993-1994, utilizando su infraestructura técnica, y la posibili- dad de compartir el avance del estudio con sus técnicos. Agradece además a Bárbara Cohen la traducción del texto original en portugués al español, y a José Sebastiảo Soares, la revisión y el trabajo gráfico. 


\section{II}

\section{Conceptualizaciones de la competitividad}

En los escritos consultados encontramos un abanico de definiciones. En un extremo de ese abanico hay definiciones que revelan mayor preocupación por los aspectos económicos de la competitividad y que hacen hincapié en sus manifestaciones más inmediatas y mensurables; en el otro extremo, hay definiciones que intentan articular los aspectos tecnoeconómicos, sociopolíticos y culturales del proceso competitivo.

No tiene sentido tratar de decidir cuales son las conceptualizaciones verdaderas o correctas, ya que todas examinan la competitividad como un fenómeno empírico a la luz de algún enfoque teorico. Lo que se podría decir es que los dos extremos del abanico de definiciones revelan intereses distintos, preocupaciones distintas, y distintas bases teóricas; tal vez uno complementa al otro. Se puede decir también que las diferencias surgen de la manera de examinar las relaciones entre desarrollo y competitividad, con enfoques que no son sólo téricos, porque incluyen estrategias, políticas y valores sociales.

\section{Conceptuallzaclón con ónfasis en la economía}

En la literatura especializada predomina el enfoque económico, con definiciones precisas y operativas, e interés por la medición cuantitativa de los componentes del proceso competitivo con miras a la utilización del método comparativo. La figura de Ricardo y las teorías sobre el comercio internacional delinean los contornos de esta manera de examinar la competitividad.

Veamos algunos ejemplos: "competitividad es la capacidad de un país, un sector o una empresa particular, de participar en los mercados externos" (Feenstra, 1989, introduccion); competitividad es la capacidad de lucrar mediante la exportación (Helleiner, 1989, p. 3); por diversas razones (análisis macroeconómico de los países, capacidad tecnológica de innovación, cualidad de los productos, etc.), "ya que estos factores son dificilísimos de medir en términos cuantitativos, tomamos la noción en términos de posiciones competitjvas relativas, claramente asociadas a los costos y precios diferenciales internacionales o, más precisamente, los cambios relativos de estos indicadores" (Durand y Giorno, 1987, p. 149); "competitividad es la habilidad sostenible de obtener ganancias y mante- ner la participación en el mercado (...) Esta definición presenta tres importantes y mensurables dimensiones: ganancias; participación en el mercado, y, a través de la palabra 'sostenibilidad', registra el aspecto temporal" (Duren, Martin y Westgren, 1992, p. 2).

Desde el ángulo económico, comprender la competitividad no exige sólo un examen de la participación en los mercados internos y externos, sino también el estudio de los precios y costos comparativos de producción, las tasas de cambio e interés, el poder de mercado y las dimensiones "no precios", como la información sobre los mercados, el diseño de los productos, el empaque, el control de calidad, la atención a los clientes, la comercialización y la distribución: incluye, en fin, la eficiencia de la economía (sector, firma, país) que exporta.

En cuanto a las políticas, los estudios indican la necesidad de relacionar el ingreso nacional marginal con el costo social marginal de un determinado bien exportable, y sus externalidades negativas o positivas (Helleiner, 1989, pp.7 y 16), es decir, las consecuencias para el bienestar o los costos no enteramente contabilizados por el sistema de precios y mercado (Bannock, Baxter y Davis, 1987).

Esta manera de emplear el concepto de competitividad en diagnósticos y pronósticos es la más usual y práctica. No hay razón para oponerse a su uso siempre y cuando las condiciones ceteris paribus y las externalidades estén claramente expuestas; por otra parte, en esta perspectiva el sistema económico se mantiene vinculado a los otros sistemas que configuran la sociedad a través del concepto de externalidad; en éste los costos sociales son sin duda considerados, aunque por ser difíciles de cuantificar, no retroalimentan la economía con informaciones fundamentales para la viabilidad, continuidad y modificación del sistema sociocultural en el cual se halla inserta.

La contaminacion industrial, el uso de las aguas de los bosques, la exclusión socioeconómica de numerosos grupos de pobres, y temas como la representación política, la democracia y los derechos humanos, no pueden, según otro grupo de cientistas sociales, ser entendidos como factores exógenos de la economía -cuando se trata de la competitividad- sino como elementos del sistema sociocultural. 
De hecho, no hay nada nuevo en esta observación crítica. Considerar las contingencias de la acción económica con respecto a la cognición, la cultura, la estructura social y las instituciones públicas tiene una larga tradición en varias disciplinas de las ciencias sociales, inclusive la economía. Parece que ahora éstas fueron "despertadas" de su descanso conceptual. Pero, más que eso, fueron llamadas a atender a nuevas situaciones empíricas (véase por ejemplo Zukin y DiMaggio, 1990). En lo que se refiere a las distintas disciplinas de las ciencias sociales, como la antropología, la economía, la política, la sociología y la historia, cabría preguntar "si existen criterios para establecer, de forma relativamente clara y sustentable", los límites entre ellas; "el análisis de los sistemas mundiales responde con un no inequívoco a esta pregunta. Todos los supuestos criterios - nivel de análisis, objeto de estudio, métodos, supuestos teóricos- carecen de validez práctica $\mathrm{o}$, si se mantienen, son obstáculos al progreso del conocimiento en vez de estímulos a su creación" (Wallerstein, 1990, pp.398-417).

El predominio de la perspectiva económica presupone que existen fuerzas manipuladas por manos "invisibles" suficientemente fuertes como para mantener, reformar y expandir el sistema sociocultural existente. Esto se podría aceptar si, de hecho, el sistema mundial funcionara "adecuadamente", como sucedió en las tres décadas después de la segunda guerra mundial. Pero ocurre que lo que distingue el sistema mundial actual es precisamente la necesidad de restablecer los supuestos básicos de su funcionamiento, como indican fuertes dosis de aleatoriedad que obligan a los agentes económicos y a los gobiernos a continuas improvisaciones. Lo que era externo a determinado subsistema "se hizo" interno, requiriendo por lo tanto una revisión de la racionalidad de la acción económica.

\section{Conceptualización con éntasis en el sistema sociocultural}

Aquí el énfasis puede estar señalado por la crítica básica a la perspectiva anterior: la conceptualización económica no se deja contaminar por las demás dimensiones del sistema social, lo que significa en la práctica la imposibilidad de formular una "estrategia integrada de reformas económicas y sociales" requeridas por una "sociedad innovadora" (Bradford, 1992, p. 18). La perspectiva sociocultural (Buckley, 1971, p. 15) parte de lo siguiente: 'Los años ' 90 constitu- yen para América Latina, años en los cuales los pobres y los gobiernos aspiran a objetivos más amplios que los que fueron factibles en los '80. Existen, ahora, nuevos imperativos para la equidad social, la competitividad internacional y la sustentabilidad ambiental, que tienen que satisfacer dentro de un contexto democrático de participación social creciente y de respeto para los derechos humanos. Para llevar a cabo estos nuevos imperativos, se debe estimular un mayor dinamismo económico y, al mismo tiempo, consolidar las conquistas recientes de estabilización y ajustes económicos" (Bradford, 1992, p.3).

Este enfoque desarrollado en los años ochenta por la CEPAL y sintetizado en buena medida en CEPAL (1990), se basa en los trabajos de Fernando Fajnzylber, y es recogido por Bradford en el Centro de Desarrollo de la Organización de Cooperación y Desarrollo Económicos (OCDE). Puede resumirse en dos puntos: i) los nuevos imperativos tecnológicos, organizacionales, institucionales, legales, políticos y culturales se imponen como elementos que prescriben el pensamiento y la acción contemporáneos, y que hacen posible diseñar una determinada configuración futura del sistema sociocultural, y ii) la competitividad gana gravitación en las relaciones que establece con otros conceptos (equidad y sustentabilidad) y valores sociales (democracia, derechos humanos y participación social) (gráfico 1).

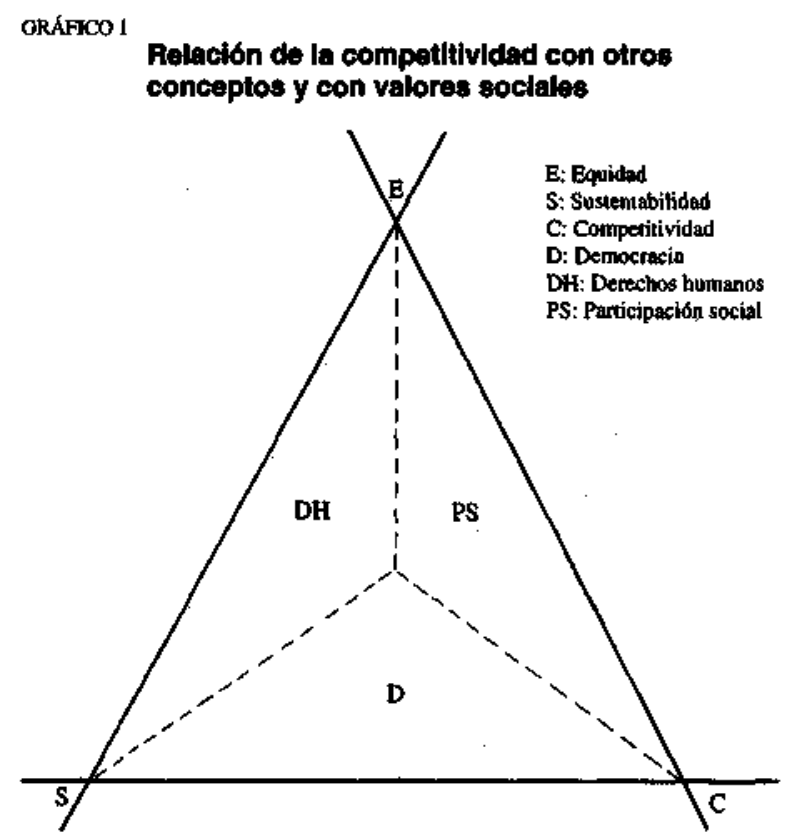

Fuente:Elaboración propia. 
Se podría decir que los autores de esta propuesta desempeñan el mismo papel que los ideólogos y los estudiosos de otras épocas. Aprovechan las experiencias de una manera crítica, cuestionan la situación vigente, se valen de las oportunidades materiales y culturales, y proponen la materialización de valores heredados de la cultura occidental. La aplicación de este modelo de desarrollo implica "el cambio social de actitudes y de comportamientos en todos los segmentos sociales rumbo a una sociedad innovadora" (Bradford, 1992, p.7).

Aunque no se puede descartar la posibilidad de una regresión civilizadora de los sistemas socioculturales, la ideología del propuesto modelo de desarrollo, que se apoya en la democracia participativa, es totalmente compatible con la competitividad basada en innovaciones continuas, o, como señaló F. Fajnzylber, la competitividad auténtica. Esto nos lleva a una pregunta decisiva, que se retoma más adelante, principalmente para quienes postulan que la competitividad es la ausencia de poder o de coerción. Además, es una visión más compleja de la determinación del proceso competitivo, ya que no destaca sólo la determinación estructural de la producción, sino que incluye, además del tema del poder, los aspectos relativos al control sobre la seguridad nacional e internacional, sobre el crédito y sobre el conocimiento, las creencias y las ideas (Strange, 1988, pp.24-29).

En este sentido, competitividad no es intercambiable con competencia; tampoco puede exhibir un status conceptual propio. De hecho, lucha por su identidad. Lucha por ser parte de un conjunto teórico, en el cual i) sea pertinente a una problemática, para la cual se presenta como una solución válida; ii) sea capaz de proveer un cuadro explicativo, y iii) sea capaz de propiciar hipotesis que se puedan simular. Además, su lucha se extiende a los ámbitos ideológico y práctico.

En el campo de la economía, se percibe la complejidad de los significados y perspectivas que establece el uso del concepto. "A pesar de la referencia obligatoria en la literatura reciente a la política industrial, al análisis del desempeño y a las perspectivas de la industria, los distintos autores no perciben la competitividad de la misma manera. Las diferencias resultan de bases teoricas, percepciones de la dinámica industrial y hasta ideologías diversas, y tienen implicaciones para la evaluación de la industria y de las propuestas de política que se formulan" (Haguenauer, 1990 , pp. 327-328).

Otros autores, después de un estudio de las defi- niciones, usos conceptuales y temas de la competitividad, concluyen que "la evaluación de la competitividad requiere una perspectiva que va más allá de los límites de la teoría de comercio tradicional para determinar el patrón de comercio y cómo éste es influenciado por la estrategia de la firma y por la intervención gubernamental. Se identificaron innumerables factores además del precio competitivo, y se presentaron distintos niveles de análisis. Vemos como inútil la investigación de un nuevo paradigma para reemplazar la teoría tradicional de comercio, pero ya se reconoció la importancia política de una nueva teoría 'estratégica' de comercio. Una importante lección que proviene de esta literatura es que sectores industriales específicos tienen importancia. Necesitamos estudios de caso detallados hechos por los estrategas de negocios para complementar los enfoques teóricamente rigurosos, basados en la teoría del equilibrio general y altamente agregados en términos macroeconómicos y de comercio" (Abbot y Bredahl, 1992, pp. 19-20). Estos autores se sienten incómodos para explicar lo que determina los patrones observados de produccion y comercio entre naciones, aunque sigue siendo clave la discusión posterior al debate sobre la paradoja de Leontief.

El examen de la competitividad desde la perspectiva de lo que llamamos sociocultural está lejos de reducirse al comercio internacional, aunque éste es parte de la competitividad.

Por más de una década, varios autores han defendido la relación explícita entre eficiencia, productividad, competitividad y el mejoramiento del nivel de vida de los ciudadanos. Porter, por ejemplo, dice: "Mi teoría comienza en las industrias y competidores individuales, y se desarrolla hasta la economía como un todo (...) La teoría que se expone en este libro pretende capturar la gran complejidad y riqueza de la competitividad actual, y no abstraerse de esto (...). Pretendo integrar los diversos elementos que influyen en el comportamiento y el crecimiento de las empresas. El resultado es un enfoque holístico, cuyo nivel de complejidad podría parecer un poco incómodo para algunas personas" (Porter, 1991, prefacio).

Vale la pena llamar la atención sobre dos ideas defendidas por éste y otros autores, que subrayan la perspectiva sociocultural: una asocia competitividad con productividad, y la otra considera la competitividad como una capacidad nacional y no de una empresa singular.

La productividad es la clave por excelencia para lograr la competitividad. En su base están las innova- 
ciones tecnológicas, organizacionales e institucionales. Las innovaciones tecnológicas, aunque no son "la causa" del desarrollo económico, se encuentran en el centro de este desarrollo (Labini, 1989, pp. 22 y 33). Cabe señalar que estas innovaciones no provienen de fuentes empíricas y aleatorias, sino de organizaciones denominadas sistemas nacionales de innovación.

Tales sistemas presentan lo que Dosi (según Villaschi, 1992, pp.51-76) identifica como tres dominios articulados: el de lo tecnológico (sistema educacional, laboratorios e investigación), el de lo económico (las formas de las unidades productivas) y el de las instituciones sociopolíticas (que facilitan u obstaculizan el desarrollo tecnologico). Por lo tanto, hay que considerar el sistema de innovaciones como un componente de los circuitos de retroalimentación del sistema sociocultural.

Para algunos autores, como Helleiner, no tiene sentido hablar de la competitividad de un país, porque de hecho quienes compiten son las empresas y los sectores económicos; los actores de la competitividad internacional se reducen a unidades y sectores productivos, y se congela todo el entorno del sistema nacional de innovaciones. Fajnzylber tiene otra visión: "En el mercado internacional compiten no sólo empresas. Se confrontan también sistemas productivos, esquemas institucionales y organizaciones sociales, en los que la empresa constituye un elemento importante, pero integrado en una red de vinculaciones con el sistema educativo, la infraestructura tecnológica, las relaciones gerencial-laborales, el aparato institucional público y privado, el sistema financiero, etc." (Fajnzylber, 1998, pp.22-23). Y para recalcar esto: "En síntesis, en el mundo actual los productos no sólo compiten, sino que en ellos se manifiesta la competencia de los sistemas productivos, tecnológicos y educacionales" (Rosales, 1990, pp. 711-712).

En el informe del seminario sobre competitividad internacional realizado en la República de Corea en abril de 1990, y coordinado por el Instituto de Desarrollo Económico del Banco Mundial, uno de los relatores, Haque, afirma que no basta entender la competitividad de los productos manufacturados por sus elementos básicos, como precio y calidad, porque en la práctica estos productos no se pueden comparar fácilmente: no hay una relación directa entre el precio y la calidad, pues es difícil especificar la calidad al diferenciarse los productos. Por otro lado, no se puede definir la competitividad sólo como la capacidad de exportar o de generar superávit comerciales, ya que se puede obtener esos resultados con medios arti- ficiales, por ejemplo, bajando la tasa de cambio o reduciendo gastos internos, como los salarios (Haque, 1991, p. 5).

Haque critica las posiciones de Porter (1991) y de Pérez (1989). La visión de Porter se basa en la inexistencia de un sistema que sea universalmente apropiado al desarrollo tecnológico, de modo que cada nación debería encontrar el suyo, a la luz de su historia, cultura y valores; sin embargo, los avances tecnológicos dictan cambios en los modos de producción y organización -algunas veces radicales-y la inadaptabilidad de uno puede frustrar la explotación del potencial del otro.

Pérez (1989) argumenta, a su vez, que los períodos de elevado crecimiento (como los años ' 50 y '60) se caracterizaron por una coherencia dinámica entre el sistema socioinstitucional y los requerimientos del cambio tecnológico, en cuanto el desajuste entre las dos esferas atraso el crecimiento en las dos últimas décadas. De acuerdo con esta visión, los países empezaron a perder competitividad internacional porque se mantuvieron aferrados a un paradigma tecnológico que dej 6 de tener validez en las nuevas condiciones. Sus dificultades provinieron, por consiguiente, de sus éxitos anteriores, porque se mantuvieron totalmente comprometidos con el paradigma por inversiones e instituciones pasadas que eran dif́ciles de cambiar o destruir. El nacimiento de un nuevo paradigma -así como ocurrió con los recientes avances en la tecnología- redefine las condiciones para la competitividad, y el éxito depende de la adaptabilidad de las instituciones nacionales. No se trata s6lo de la cuestion de crear nuevas industrias y productos en perjuicio de las antiguas.

Según Haque, no obstante, el problema con este enfoque es que aunque hace hincapié en la armonización entre tecnología e instituciones, no logra explicar el éxito de los países de industrialización reciente que superaron las fuentes tradicionales en la producción manufacturera (Haque, 1991, pp.6 y 7).

Otro relator del Seminario dejo en claro la perspectiva sociocultural, mostrándose firme en su opinión de que "la competitividad nacional no es sencillamente un fenómeno económico ni un fenómeno impulsado por el mercado. La eficiencia pasiva (esto es, la que acepta los precios como correctos con la expectativa de que la competitividad se adapte automáticamente) no es la experiencia de páses como Corea, que ha tenido un buen desempeño en la economía mundial. Leyes, costumbres, lenguaje, hábitos en los negocios y otras peculiaridades nacionales des- 
empeñan un papel importante en la determinación de la competitividad y del comercio. Es necesario tener una perspectiva amplia y globalizadora de la sociedad" (Bradford, 1991, pp. 18).

Este enfoque se basa en la competitividad nacional; señala la relación contradictoria entre instituciones nacionales, creadas para difundir en el país un paradigma tecnoeconómico de carácter mundial; considera la experiencia histórica de los países asiáticos recién industrializados, y concluye que las estrategias y políticas nacionales son necesarias para crear las fuentes de competitividad.

\section{Una evaluación preliminar}

Definir la competitividad como la participación en el mercado es un buen comienzo; ampliarla con la incorporación de la estructura y la conducta de las empresas y de los sectores económicos es un buen avance, y sin duda, algo muy útil para hacer diagnósticos tecnoeconómicos. La posibilidad de explorar más el concepto de externalidades (positivas y negativas) a la luz del nuevo paradigma de producción flexible, junto con muchas otras contribuciones teóricas de la economía industrial y de los negocios estratégicos, permite pensar en avances de importancia en una perspectiva más específicamente económica de la competitividad. Esto haría difícil incorporar las otras dimensiones del sistema social, hoy tan relevantes como la económica, en la perspectiva económica de la competitividad.

Por otro lado, el concepto de competitividad visto desde el ángulo sociocultural es excesivamente extenso, aunque está articulado a una red conceptual (también en busca de su status teórico) que implica la pérdida de su carácter operativo; sus límites son muy tenues y su estructura interna muy fluida. La competitividad aparece aquí como una mezcla de: i) una visión del mundo contemporáneo, sumergido en valores sociales, que acepta diversas combinaciones de organización y mercado; ii) conceptos de distintas vertientes teóricas que, al reunirse (lo que está todavía poco claro), sobrepasan el alcance de otros conceptos utilizados en teorías como la del comercio internacional; iii) vida práctica, donde se lucha ferozmente por mercados y donde no hay regulaciones internacionales tan "civilizadas" que permitan a los agentes experimentar los valores sociales propuestos por el modelo. De hecho, esta conceptualización de competitividad pretende, junto con los conceptos de equidad y sustentabilidad, apuntar hacia el futuro, como un modelo, y disponer de capacidad prescriptiva sobre las acciones presentes.

Por eso sugerimos que se considere la competitividad como un mapa, con territorios y caminos, que frente a preguntas particulares permite elaborar un concepto adecuado. Esto porque la competitividad se ha convertido, principalmente después de la crisis de 1979-1982, en una de las principales "normas" del inestable juego internacional. La apertura comercial, los ajustes estructurales, la reconversión productiva, la coexistencia inteligente con los recursos naturales, la lucha contra la pobreza, en fin, prácticamente todo se enfoca, de una manera u otra, a través del prisma de la competitividad. Lo que la ha transformado -en un clima conceptual amplio y ambiguo- en una especie de principio obligado de evaluación internacional para los organos públicos y privados (incluidos por cierto los organismos crediticios) y ha repercutido en la formulación y aplicación de las estrategias empresariales y políticas nacionales.

La polémica entre el Banco Mundial y Japón, al principio de los años noventa, puso de relieve que la cuestión no residía en estar a favor o en contra de la apertura de las economías nacionales, sino en la manera de implementarla. Mientras el presidente del Banco Mundial afirmaba en febrero de 1990 que las "fuerzas del mercado y la eficiencia económica fueron (en la década pasada) los mejores caminos para alcanzar el tipo de crecimiento que es el antídoto de la pobreza" (Broad y otros, 1990-1991, p.144), Japón sostenía que "en la década de los 80 , tanto la teoría como la política economica estuvieron fuertemente orientadas hacia la búsqueda de la eficiencia. En este sentido, fue un período único. Sin embargo, este período llego a su fin. Lo que se necesita ahora es una política bien equilibrada entre la eficiencia y la equidad, para promover el bienestar de toda la sociedad. El enfoque de ajuste estructural del Banco Mundial debería transformarse para reflejar este cambio de rumbo" (Japón, 1991).

Hoy al parecer se dispone de una experiencia razonable para adoptar una posición más madura frente a las estrategias simplistas defendidas por algunos organismos intemacionales durante los años ochenta. Estrategias según las cuales bastaba con abrir los mercados y lanzar los países a la exportación para alcanzar el crecimiento, ya no son defendidas ni siquiera por los que las formularon (el Banco Mundial, incluidas la Corporación Financiera Internacional (CFI) y la Asociación Internacional de Fomento (AIF). Además, se consideran falsos dilemas las dicotomías rígidas 
que llevaban a la creación de extrafias oposiciones, como Estado fuerte contra Estado mínimo, economía de mercado contra economía de intervención y sustitución de importaciones contra promoción de exportaciones.

Un estudio de lo sucedido en la práctica revela que, a pesar de que la competitividad está implícita en la liberalización de las economías nacionales, de hecho se mantienen los esquemas proteccionistas y de incentivos a través de los cuales todos los países tratan de alcanzar sus objetivos nacionales. Es más: la desregulación y la privatización no han eliminado el papel del Estado en el desarrollo económico, porque no se han creado instituciones capaces de dar el necesario apoyo a las innovaciones, al comercio intemacional, a las inversiones transnacionales y a la negociaciones bilaterales y multilaterales, sobre todo en los países en desarrollo.

\section{IV}

\section{Un mapa de la competitividad}

El mapa de la competitividad tiene dos polos: el poder estructural mundial y el desarrollo nacional/regional de los países o regiones que buscan el crecimien- to y el desarrollo a través de su integración en el mundo (gráfico 2).

El concepto de poder constituye un elemento vi-

\section{GRÁFICO 2 \\ Mapa de la compotitlvidad}
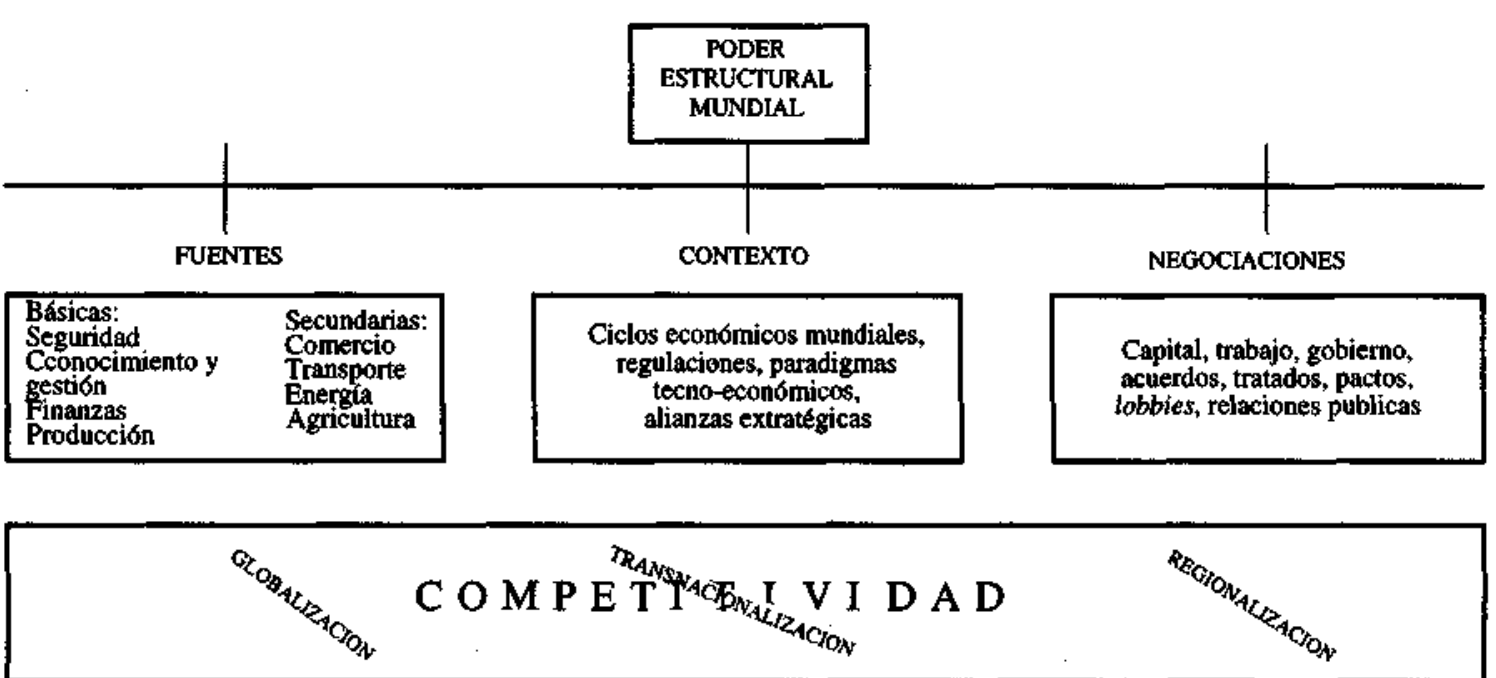
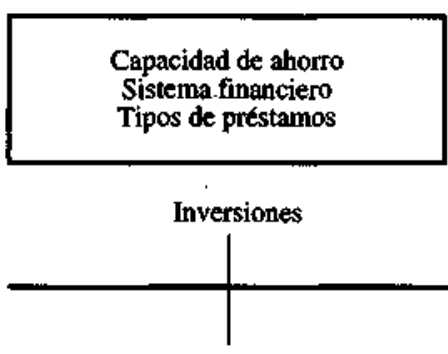

Fuente: Elaboracion propia.

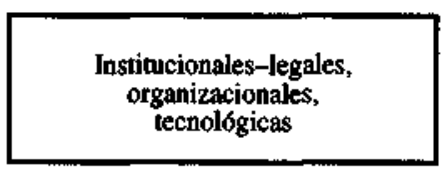

Innovaciones
Lobbies, relaciones publicas, acuerdos, tratados, pactos, capital, trabajo, gobiemo

\section{Negociaciones}


tal del mapa de la competitividad. El contexto en que ocurre y se estructura la competitividad, y también sus fuentes, se mezclan y se cruzan con los componentes de poder; de ahí deriva la inclusión del concepto amplio de negociación, suponiendo que no hay poder sin negociación de algún tipo.

"Es imposible estudiar economía política y especialmente economía política internacional sin prestar atención especial al papel del poder en la vida económica (...) Cada sistema (económico político nacional) refleja una mezcla distinta en la ponderación de (los valores básicos) riqueza, orden, justicia y libertad. Lo que decide la naturaleza de la mezcla es, fundamentalmente, una cuestión de poder" (Strange, 1988 , p. 23). El poder y la competitividad están muy relacionados, en buena medida porque comparten las mismas fuentes básicas: la seguridad, el conocimiento, las finanzas y la producción (véase Müller, 1992, pp. 12-25).

Prestar atención a las fuentes de poder en un contexto específico es sólo parte del proceso competitivo. Ir más allá significaría examinar las negociaciones claves de cada situación particular, una vez que existieran las condiciones para decidir lo que se puede o no cambiar. Una negociación de los productores brasileños con asociaciones de consumidores de jugo de naranja estadounidenses en torno a ciertas características del producto, con miras a la competencia en el mercado de Estados Unidos, puede convertirse en un momento dado en el elemento decisivo para el éxito de una estrategia competitiva brasileña.

"Poder estructural mundial" es aquel poder que forma las estructuras tecnoeconómicas y sociopolíticas globales, y que decide cómo deben operar los demás Estados, instituciones, empresas y procesos económicos (Strange, 1988, pp.24-29). Se puede identificar a los que poseen este poder por su papel protagónico en el mundo contemporáneo: Alemania, Canadá, Estados Unidos, Francia, Italia, Japon y el Reino Unido (el G-7); América del Norte, Europa occidental y Japón (la Tríada, según la expresión de Ohmae, 1985); China y Rusia; las empresas transnacionales; el FMI y el Banco Mundial; la OTAN y el Pentágono - para citar sólo algunos ejemplos (véase en Plano y Olton, 1982).

Es tan importante saber quién participa del poder estructural como saber de qué manera participa, es decir, ¿cuáles son las fuentes de poder? $\mathrm{Y}$ respecto de la competitividad: ¿cuáles son las fuentes de la capacidad competitiva? Aquí se podría decir que la competitividad es una expresión del poder estructu- ral, o un efecto combinado del control sobre las fuentes de poder unido a la capacidad de manejar las negociaciones claves en un contexto determinado. $Y$ más aún, habría que considerar el efecto retroalimentador que la competitividad tiene sobre el poder estructural, sin que un concepto se identifique con el otro.

El mapa indica que la competitividad permea todos los procesos, constituyéndose en una de las maneras en que los países desarrollados se conectan con los que buscan el desarrollo, a nivel nacional o regional. La búsqueda, el mantenimiento y la expansión de la competitividad de los países desarrollados y los en desarrollo deben tomar en cuenta tres tendencias básicas a nivel internacional, que sirven de referencia para las estrategias: la globalización, la transnacionalización y la regionalización (SELA, 1991, pp. 20-24). El núcleo del poder estructural —es decir, aquel conjunto de actores que ejercen la hegemonía (la Tríada, por ejemplo) - se presenta como una articulación de los Estados nacionales que poseen las más avanzadas fuentes de poder y de competitividad. Las tres tendencias señaladas indican las condiciones que se imponen a empresas, sectores, economías y regiones para poder integrarse dinámicamente en el mundo contemporáneo.

En este contexto surge la tendencia a revisar el papel del Estado nacional en el mercado mundial, y por ende del Estado en la nación, debido a la presencia de la globalización y la transnacionalización. Esta tendencia es contrarrestada por la aparición de agrupaciones regionales de países (regionalización). En otras palabras, hay factores que erosionan la capacidad de autonomía de los Estados nacionales para controlar su demanda global y poner en práctica estrategias y polf́ticas dirigidas a los objetivos nacionales. $Y$ a la vez, la tendencia contraria ya no se manifiesta tan sólo en una estrategia para restablecer la fortaleza de los Estados nacionales, sino que busca organizar un ente supranacional, es decir, un ente regional.

Uno de los factores que erosionan la capacidad de autonomía de los Estados nacionales es la transnacionalización. Esta resulta de la acción de empresas que tienen estrategias, controles, inversiones y gestiones organizados en redes que se complementan, que se extienden por todo el planeta y que buscan optimizar la productividad y las ganancias. Cabe destacar que en el centro de este proceso ya no están las empresas multinacionales de las décadas de 1950 , 1960 y 1970 , sino las organizaciones transnacionales (Wendt, 1993).

El otro factor es la globalización. Esta emana de 
la mayor apertura de las economías nacionales, del aumento de la participación de los flujos y acervos externos en los internos, ya sea de carácter productivo, mercantil, financiero o de servicios. En este sentido, la globalización incluye la transnacionalizacion; además, lleva a utilizar el concepto de interdependencia de intereses, contrario al de subordinación o de imperialismo. ${ }^{1}$

La regionalización o la formación de bloques entraña una reformulación de la distribución del poder entre los países de un mismo bloque, y entre bloques. El ejemplo más avanzado de regionalización es la Unión Europea; la mayoría de los otros bloques constituyen, de hecho, una coordinación comercial de las antiguas áreas de influencia, que podrían o no ser convertidas en bloques económicos.

Las negociaciones claves a nivel nacional o regional son fundamentales para una regulación que facilite un mayor control del circuito de indeterminacion socioeconómico. Como ejemplo, basta citar las alianzas entre los grandes actores del sistema sociocultural: los empresarios, los trabajadores y los gobiemos.

La negociaciones a nivel internacional son decisivas para la integración competitiva. Acuerdos, tratados, pactos y grupos de presión (lobbies) constituyen mecanismos indispensables para la competitividad intemacional. Según Kramer, uno de los aspectos menos estudiados del éxito de los "tigres asiáticos" es la hábil gestión política de sus relaciones comerciales con los Estados Unidos, a través de un versátil y sofisticado lobby en Washington. Hace poco, Taiwán hizo la proeza de mantenerse como el principal beneficiario del Sistema Generalizado de Preferencias (SGP), después de los esfuerzos contrarios de la burocracia de comercio estadounidense, de ajustarlo en cada nueva ronda de revisión del SGP. Datos de 1983 del Departamento de Justicia estadounidense muestran que la República de Corea gasto $\mathbf{4 . 5}$ millones de dolares en 47 superabogados o expertos en relaciones públicas, y la provincia china de Taiwán, 2.5 millones en 39 expertos en relaciones públicas. También se han formado coaliciones con grupos de intereses complementarios existentes en la sociedad americana. Como las medidas proteccionistas aumentan los precios y perjudican la calidad de los productos, los coreanos y taiwaneses, siguiendo el camino pionero

\footnotetext{
1 Véase un enfoque crítico de la interdependencia, sobre todo en las relaciones Norte-Sur, de la competitividad como la nueva ideología; de la lógica de la guerra, y de las contradicciones de la globalización, en Manière de voir, 1993.
}

de los fabricantes de automoviles japoneses, crearon alianzas con grupos defensores del consumidor y representantes de cadenas de venta al por menor (Kramer, 1991, pp.41-42).

Vale la pena señalar lo que se podría llamar un "modelo mundial" en formación, y to que éste promete. Con las transformaciones geopolíticas e ideológicas, unidas a los cambios en las estrategias de competitividad y de productividad, se han planteado nuevos desafíos a la regulación mundial y a las regulaciones nacionales. Estos desafíos se dan en un contexto frágil, en el cual hay continuas improvisaciones para encarar los problemas. Es difícil, por lo tanto, generalizar un modelo de desarrollo que está en construcción, un modelo inacabado, porque si bien a nivel microeconómico y sectorial están delineadas las trayectorias de lo que se debe hacer, a nivel macroeconómico -que incluye, entre otros aspectos la seguridad social y el desempleo- no ocurre lo mismo. Las principales dificultades residen en la generalización de las promesas contenidas en el modelo: participación social, equidad, y control del medio ambiente. Son dificultades para los países desarrollados y, principaimente, para los países en desarrollo, ya que envuelven cambios profundos en el ambiente interno.

Las relaciones entre el poder estructural mundial y el desarrollo que buscan, por ejemplo, los países de América Latina y el Caribe, se dan en un "campo de fuerzas" competitivo, en el cual se entrelazan tres grandes "corrientes de fuerza": la globalización, la transnacionalización y la regionalización (gráfico 2). Los países que tienen el poder son los líderes en estas corrientes. Así, y vista desde la perspectiva de la región, la competitividad sería la capacidad de un pais de adaptarse a la estructura del poder mundial, a través de la participación en las determinaciones de índole global, nacional y regional. Esta capacidad se puede convertir en realidad, no como en el modelo de industrialización "hacia adentro", sino mediante el ingreso a la nueva división del trabajo centrada en la especialización subsectorial, lo cual favorece el comercio y las inversiones intrasectoriales que son "el núcleo de la teoría moderna de comercio" (Cohen, 1992, p.35).

Para participar en el "campo de fuerzas" competitivo, los países en desarrollo disponen de tres subsistemas: el de inversiones, el de innovaciones y el de negociaciones. No es que cada país de la región deba contar con los tres subsistemas, sino que debe crear la capacidad nacional de articular el subsistema que tiene (o una parte) de él con los que están disponibles 
en otros parses de la región. El desarrollo de los servicios de comunicación instantánea y de redes de procesamiento de informaciones permiten el "comercio" intrasectorial internacional.

El objetivo de lograr una sociedad innovadora en la región, competitivamente integrada en el mundo, está ligado a la capacidad de efectuar innovaciones, no sólo tecnológicas, sino también organizacionales e institucionales y legales. El conocimiento de este subsistema, junto con el conocimiento del paradigma tecnoeconómico predominante en las economías nacionales, facilita la selección de las innovaciones necesarias para una o varias de las estrategias competitivas que se piensa aplicar. Lo importante es que las innovaciones se conviertan en el motor básico para conseguir los objetivos de la competitividad; ellas serán los parámetros dentro de los cuales se buscaría adecuar los subsistemas de inversiones y de negociaciones claves.

Hay que recordar que los mecanismos de protección y los incentivos temporales para productos, empresas y sectores destinados a crear y expandir capacidades competitivas nacionales o regionales, aunque en principio son contradictorios al espíritu de apertura de las economías (globalización y transnacionalización), no son antagónicos a las fuerzas que gobiernan la competitividad internacional -con la condición, que al parecer va a predominar- de que estos mecanismos sean negociados interna y externamente.

Por último, en el orden interno de cada país de la región se hace cada vez más difícil continuar con un modo de producción que destruye el medio ambiente, y con un modo social de organización que acentúa las asimetrías entre los grupos sociales a través de la concentración de la riqueza y el aumento de la pobreza. Hoy, esta perversa polarización se considera un obstáculo al crecimiento económico: sobre todo la pobreza, entendida como la ausencia de mercado y la carencia de poder social. Para aplicar innovaciones tecnológicas y de organización que aumenten la productividad y consoliden un patrón competitivo auténti$c 0$, se requiere la redistribución del poder en las sociedades nacionales y la incorporación a él de los sectores pobres e indigentes. Hoy es imperativo lograr la reorganización sociopolítica, articulada con innovaciones para lograr el desarrollo nacional/regional en un contexto mundial competitivo.

\section{Bibliografía}

Abbot, P. y M. Bredahl (1992): Competitiveness: definitions, useful concepts and issues, trabajo presentado al Symposium on Competitiveness in international food markets, Annapolis, Maryland, Estados Unidos, 7 y 8 de agosto.

Bannock, G., R. Baxter y E. Davis (1987): Dictionary of Economics, Londres, Penguin Books, 4 ' edición.

Bradford, Colin (1991): International competitiveness. Interaction of the public and private sectors, Collected papers from EDI policy seminar, Washington, D.C., Banco Mundial.

(1992): Toward an integrated pollcy framework, Paris,

OECD Development Center, invierno, Organización de Coopera-

ción y Desarrollo Económicos (OCDE).

Broad, Robin, J. Cavanagh y W. Bello (1990-91): Development: the market is not enough, Foreign Policy, $\mathbf{N}^{\circ} 81$, Washington, D.C., Carnegie Endowment for International Peace.

Buckley, Walter (1971): A sociologia e a moderna teoria dos sistemas, São Paulo, Brasil, Cultrix/usp.

CEPAL (Comisión Económica para America Latina y el Caribe) (1990): Transformación productiva con equidad (LC/G. 1601-P), Santiago de Chile. Publicación de las Naciones Unidas, $\mathbf{N}^{\circ}$ de venta S.90.II.G.6.

Cohen, Stephen S. (1992): As opçס̃es da Europa no novo cenário econômico, Novos estudos CEBRAP, $\mathbf{N}^{\circ} 35$, São Paulo, Centro Brasileño de Análisis y Planeamiento, marzo.

Durand, M. y C. Giomo (1987): Indicators of international competitiveness: conceptual aspects and evaluation, $O E C D$ Economic Studies, $N^{\circ}$ 9, París, Organización de Cooperación y Desarrollo Económicos (OCDE).

Duren, E., L. Martin y R. Westgren (1992): A framework for as- sessing national competitiveness and the role of private strategy and public policy, trabajo presentado al Symposium on Competitiveness in international food markets, Annapolis, Maryland, Estados Unidos, 7 al 8 de agosto.

Fajnzylber, Fernando (1988): Competitividad internacional: evolución y lecciones, Revista de la CEPAL, N $\mathrm{N}^{\circ} 6$ (LC/G. 1537-P), Santiago de Chile, CEPAL.

Feenstra, R. (1989): Trade Policies for Intemational Competitiveness, Chicago, Ill., Estados Unidos, The University of Chicago Press.

Haguenauer, L. (1990): Competitividade. Uma resenha da bibliografia recente com ênfase no caso brasileiro, Pensamiento iberoamericano, $\mathrm{N}^{*} 17$, Madrid, CEPAL/Instituto de Cooperación Theroamericana (ICI/Sociedad Estatal Quinto Centenario.

Haque, Irfan ul (1991): International competitiveness. Interaction of the public and private sectors, Collected papers from EDI policy seminar, Washington, D.C., Banco Mundial.

Helleiner, G. (1989): Increasing international competitiveness: a conceptual framework, Lectures Notes, Barbados, mimeo.

Japon (1991): Temas relacionados con el enfoque del Banco Mundial sobre ajuste estructural. Propuesta de un socio mayoritario (Japón), Fondo de Cooperación Económica a Ultramar (OECF), octubre. Traducido por la Secretaría Técnica para la Reforma del Estado Costarricense, julio de 1992.

Kramer, Paulo (1991): Brasil-EE.UU.: problemas de "lobby", comunicação, RP, Sdo Paulo em perspectiva, vol. $5, \mathrm{~N}^{\circ} 3$ Săo Paulo, Fundação SEADE.

Labini, P. Sylos (1989): Nuove tecnologie e disoccupazione, Roma-Bari, Sagitari Laterza. 
Manière de voir (1993): Les frontières de l'economie globale. Le monde diplomatique, $\mathrm{N}^{\circ} 470,40$ anné, Parrs.

Mtiller, Geraldo (1992): Competitividade e negociacöes no complexo agroindustrial brasileiro, Rascunho, $\mathrm{N}^{\circ} 20$, Rio Claro, Brasil, Universidade Estadual Paulista Julio de Mesquita Fillho (UNESP)/IGCE/DPR.

Ohmae, Kenichi (1985): Triad Power. The Coming Shape of Globat Competition, Nueva York, The Free Press.

Pérez, Carlota (1989): Cambio tecnico, reestructuración competitiva y reforma institucional en los países en desarrollo, El trimestre económico, vol. LIX (1), No 233, México, D.F., Fondo de Cultura Econónica.

Plano, J. y R. Olton (1982): The International Relations Dictionary, Santa Bárbara, California, Estados Unidos, ABC-CuO, $3^{*}$ edición.

Porter, Michael (1991): La ventaja comparativa de las naciones, Buenos Aires, Javier Vergara Editor S.A.

Possas, Mario (1985): Estruturas de mercado em oligopdilo, Saro Paulo, Hucitec.

Rosales, Osvaldo (1990): Competitividad, productividad e inserción externa de America Latina, Comercio exterior, vol. 40 ,
N 8, México, D.F., Banco Nacional de Comercio Exterior, S.N.C.

SELA (Sistema Economico Latinoamericano) (1991): Desarrollo industrial y cambio tecnologico, Caracas, SELAEditorial Nueva Sociedad.

Strange, Susan (1988): States and Markets, Londres, Pinter Publishers.

Tannanes, Ramón (1988): Diccionario de economia, Madrid, Alianza Editorial.

Villaschi, Arlindo (1992): O Brasil e o novo paradigma tecnológico de desenvolvimento economico mundial, Ensaios FEE, No 13, Porto Alegre, Brasil.

Wallerstein, Immanuel (1990): Análisis de los sistemas mundiales, en Anthony Giddens y Jonathan Turner (eds.), La teoría social hoy, Madrid, Alianza Universidad. Versión española de Jesús Alborés.

Wendt, Henry (1993): Global Embrace. Corporate Challenges in a Transnational World, Nueva York, Harper Business.

Zukin, S. y P. Dimaggio (eds.) (1990): Structures of Capital. The Sacial Organization of the Economy, Nueva York, Cambridge University Press. 